\title{
Protein Markers of Neurotransmitter Synthesis and Release in Postmortem Schizophrenia Substantia Nigra
}

\author{
Kirsten E Schoonover, ${ }^{*}$, Lesley A McCollum² and Rosalinda C Roberts ${ }^{2}$ \\ 'Department of Psychology and Behavioral Neuroscience, University of Alabama at Birmingham, Birmingham, AL, USA; ${ }^{2}$ Department of Psychiatry \\ and Behavioral Neurobiology, University of Alabama at Birmingham, Birmingham, AL, USA
}

\begin{abstract}
The substantia nigra (SN) provides the largest dopaminergic input to the brain, projects to the striatum (the primary locus of action for antipsychotic medication), and receives GABAergic and glutamatergic inputs. This study used western blot analysis to compare protein levels of tyrosine hydroxylase (TH), glutamate decarboxylase (GAD67), and vesicular glutamate transporters (vGLUTI and vGLUT2) in postmortem human SN in schizophrenia subjects $(n=13)$ and matched controls $(n=12)$. As a preliminary analysis, the schizophrenia group was subdivided by $(1)$ treatment status: off medication $(n=4)$ or on medication $(n=9)$; or $(2)$ treatment response: treatment resistant $(n=5)$ or treatment responsive $(n=4)$. The combined schizophrenia group had higher TH and GAD67 protein levels than controls (an increase of 69.6\%,P $=0.01$ and 19.5\%, $P=0.004$, respectively). When subdivided by medication status, these increases were found in the on-medication subjects (TH 88.3\%, $P=0.008$; GAD67 40.6\%, $P=0.003$ ). In contrast, unmedicated schizophrenia subjects had higher vGLUT2 levels than controls (an increase of $28.7 \%, P=0.04 \mathrm{I}$ ), but vGLUT2 levels were similar between medicated schizophrenia subjects and controls. Treatment-resistant subjects had significantly higher TH and GAD67 levels than controls (an increase of I21.0\%, $P=0.0003$ and $58.7 \%, P=0.004$, respectively). These data suggest increases in dopamine and GABA transmission in the SN in schizophrenia, with a potential relation to treatment and response. Neuropsychopharmacology (2017) 42, 540-550; doi:I0.1038/npp.2016.164; published online 2I September 2016
\end{abstract}

\section{INTRODUCTION}

Schizophrenia is a severe life-changing disease with complicated biological alterations. Elevated striatal dopamine (Meyer-Lindenberg et al, 2002; Hietala et al, 1995; Howes and Kapur, 2009; see as review Perez-Costas et al, 2010) has been suspected to play a role in schizophrenia pathology since the development of antipsychotic drugs in the 1950s (Delay et al, 1952) and the discovery that the majority of neuroleptics block striatal dopaminergic $\mathrm{D}_{2}$ receptors (Carlsson and Lindqvist, 1963; Creese et al, 1976). The main sources of these striatal inputs are the dopamine neurons in the substantia nigra (SN) and ventral tegmental area (VTA) (Fallon and Moore, 1978). Despite this relationship and the importance of dopamine in schizophrenia, few studies have examined the SN in schizophrenia. Some postmortem studies indicate increased dopamine activity, protein, and mRNA levels (Howes et al, 2013; Mueller et al, 2004; Toru et al, 1988), whereas others do not (Perez-Costas et al, 2012; Ichinose et al, 1994). Imaging studies report disturbed functional connectivity (Hadley et al, 2014) that is correlated with increased dopamine synthesis capacity (Howes et al,

*Correspondence: KE Schoonover, Department of Psychiatry and Behavioral Neurobiology, University of Alabama at Birmingham, Sparks Center 866, 1720 2th Avenue South, Birmingham, AL 35294, USA, Tel: + I 304437 4554, Fax: + I 205996 9377, E-mail: Kschoon I@uab.edu Received 29 April 20 16; revised I 5 July 20 16; accepted 13 August 20 16; accepted article preview online 23 August 2016
2013; Watanabe et al, 2014) and symptom severity (Yoon et al, 2014).

SN dopamine neurons receive multiple types of input, such as glutamatergic and GABAergic afferents (see as review Perez-Costas et al, 2010). The glutamate and GABA systems play a regulatory role in the SN dopaminergic system exciting or inhibiting the dopamine neurons, respectively. An excess of glutamate and/or a deficit of GABA could disrupt the excitatory balance of the $\mathrm{SN}$, resulting in the dopaminergic hyperactivity mentioned above. The current postmortem study seeks to elucidate the roles of dopamine, GABA, and glutamate in the SN in schizophrenia. We hypothesize possible mechanisms that underlie hyperexcitability in the SN could be increased glutamate and/or decreased GABA levels. Thus, we measured (1) tyrosine hydroxylase $(\mathrm{TH})$, the rate-limiting enzyme in dopamine biosynthesis (Nagatsu et al, 1964); (2) the vesicular glutamate transporters (vGLUT1 and vGLUT2) that are responsible for packaging glutamate into vesicles (Bellocchio et al, 2000) for cortical (vGLUT1) and subcortical (vGLUT2) release (Lavoie and Parent, 1990); and (3) glutamic acid decarboxylase (GAD67) that synthesizes GABA from glutamate (StormMathisen, 1974). As there are biological correlates to medication status and treatment response, we also performed exploratory analyses of treatment status and response. Taken together, these markers of neurotransmitter signal transduction and synthesis capacity may reveal the underlying mechanisms of the abnormalities seen in 
schizophrenia. These data have been published previously in abstract form (Schoonover et al, 2015).

\section{MATERIALS AND METHODS}

\section{Postmortem Brains}

Human brains were obtained from the Maryland Brain Collection with consent from the next of kin with IRBapproved protocols. These cases were obtained in collaboration of the Office of the Chief Medical Examiner (OCME) in Baltimore, Maryland. The schizophrenia cohort was tested as a whole and then divided by treatment status or treatment response. These subjects were all different cases than we have previously studied (Perez-Costas et al, 2012; Rice et al, 2016). Schizophrenia cases $(n=13)$ were compared with matched normal controls (NCs, $n=12$ ). As a preliminary investigation, the schizophrenia group was then subdivided by treatment status: no medication (SZ-Off, $n=4$ ) or on medication (SZ-On, $n=9$ ); or treatment response: treatment resistant (TR, $n=5$ ) and treatment responsive (RESP, $n=4$ ). Cases were selected based on the best match of the demographic factors age, race, sex, postmortem interval (PMI), sample $\mathrm{pH}$, and number of years frozen (Table 1). Exclusionary criteria for both schizophrenia subjects and controls were: history/evidence of intravenous drug abuse, HIV/AIDS, hepatitis B, head trauma, comorbid neurological disorders, custodial death, victims of fire, unknown next of kin, children, or decomposed subjects. In addition, comorbid mental illness in schizophrenia subjects and history of serious mental illness for NCs were exclusion factors. Normal controls died from the following causes: gunshot wounds, aneurysm, motor vehicle accident, and a cardiac event; schizophrenia cases died from: a gastrointestinal bleed, suicide, a cardiac event, tuberculosis, a seizure, fatty liver, asphyxiation, and antidepressant intoxication. However, agonal status has not been shown to have any effect on protein (Stan et al, 2006), and therefore is not an issue with the current investigation.

Diagnosis of schizophrenia was confirmed independently by two psychiatrists based on DSM criteria at the time of diagnosis (DSM-III-R through DSM-IV-TR) using the
Structured Clinical Interview for the DSM (SCID). Subject clinical information (such as neuroleptic compliance, age of disease onset, symptomology, treatment response) was obtained from autopsy and medical records, in addition to family interviews. Furthermore, we had access to toxicology analysis performed by the OCME on all subjects. All but one subject had negative drug test results: one schizophrenia patient tested positive for cocaine at the time of death. Postmortem cases were characterized using treatmentresistant criteria (Conley and Kelly, 2001; Kane et al, 1988) as done previously (Roberts et al, 2009, 2012); in this particular cohort, there was enough information to classify 9 of the 13 subjects. Placement into the off-medication schizophrenia group required being off any antipsychotic medication for a minimum of 6 months before death.

\section{Western Blotting}

Tissue and protein preparation. The SN was blocked in a similar way as in Perez-Costas et al (2012) using the criteria of Damier et al (1999). Location of the caudal SN is described by Figure 8 in Damier et al (1999) and Figure 2 in Hall et al (2014). Briefly, in coronal sections of the SN, rostral SN sections were at the level of the red nucleus; middle sections were at the level of the red nucleus and third nerve rootlet fibers; the caudal sections were caudal to third nerve rootlets. The blocks were further trimmed to a rectangular shape to remove as much excess nonnigral tissue. A perimeter of $\sim 2 \mathrm{~mm}$ of nonnigral tissue remained in these blocks (Figure 4c). Frozen caudal SN was sectioned into 3 series (16 $\mu \mathrm{m}$ sections) and alternatively collected in vials. Series 2 of the sectioned tissue was sonicated in lysis buffer $(500 \mu \mathrm{l} / 0.1 \mathrm{~g}$ of human tissue) containing Tris-HCL $(\mathrm{pH}$ 8.0), EDTA, sodium chloride, sodium dodecyl sulfate, and a protease inhibitor cocktail (Sigma; P8340). Tissue homogenate was centrifuged at 13500 r.p.m. for $15 \mathrm{~min}$ at $4{ }^{\circ} \mathrm{C}$. Supernatant (total cell lysate) was then extracted and protein concentration determined via the Lowry method (Bio-Rad, Hercules, CA; 500-0113, 500-0114).

Gel electrophoresis and western blotting. Western blots were used to measure protein levels of TH, GAD67,

Table I Demographics and Markers of Tissue Quality

\begin{tabular}{|c|c|c|c|c|c|c|c|c|c|}
\hline & No. & Age, years & PMI, h & $\mathrm{pH}$ & Years frozen & Age of onset & DUI, years & Race & Sex \\
\hline NC & 12 & $50.4 \pm 15.7$ & $15.3 \pm 6.8$ & $6.6 \pm 0.4$ & $17.2 \pm 4.5$ & NA & NA & $9 \mathrm{C} / 3 \mathrm{AA}$ & $9 M / 3 F$ \\
\hline SZ & 13 & $42.2 \pm 13.1$ & $14.2 \pm 9.3$ & $6.6 \pm 0.4$ & $14.4 \pm 3.2$ & $22.9 \pm 5.4$ & $18.6 \pm 9.8$ & $8 \mathrm{C} / 5 \mathrm{AA}$ & $10 M / 3 F$ \\
\hline t-test & & $P=0.17$ & $P=0.76$ & $P=0.84$ & $P=0.26$ & NA & NA & $\chi^{2}=0.47$ & $\chi^{2}=0.91$ \\
\hline TR & 5 & $38.4 \pm 12.3$ & $\mid 3.8 \pm 8.1$ & $6.7 \pm 0.3$ & $15.2 \pm 3.5$ & $18.0 \pm 5$ & $24.0 \pm 9.5$ & $3 \mathrm{C} / 2 \mathrm{AA}$ & $3 \mathrm{M} / 2 \mathrm{~F}$ \\
\hline RESP & 4 & $38.8 \pm 7.5$ & $18.0 \pm 12.4$ & $6.6 \pm 0.4$ & $13.5 \pm 2.6$ & $25.0 \pm 2.6$ & $16.3 \pm 11.2$ & $2 \mathrm{C} / 2 \mathrm{AA}$ & $3 M / I F$ \\
\hline SZ-On & 9 & $38.6 \pm 9.8$ & $15.7 \pm 9.8$ & $6.6 \pm 0.3$ & $17.2 \pm 2.3$ & $21.5 \pm 5.2$ & $20.2 \pm 10.2$ & $5 \mathrm{C} / 4 \mathrm{AA}$ & $6 \mathrm{M} / 3 \mathrm{~F}$ \\
\hline SZ-Off & 4 & $50.3 \pm 17.5$ & $11.0 \pm 8.6$ & $6.4 \pm 0.4$ & $21.8 \pm 3.0$ & $27.0 \pm 4.2$ & $14.0 \pm 9.9$ & IC/3AA & $4 \mathrm{M} / \mathrm{OF}$ \\
\hline ANOVA/t-test & & $P=0.16$ & $P=0.62$ & $P=0.77$ & $P=0.11$ & $P=0.23$ & $P=0.49$ & $\chi^{2}=0.31$ & $\chi^{2}=0.19$ \\
\hline
\end{tabular}

Abbreviations: DUI, duration of illness; NC, normal controls; PMI, postmortem interval; RESP, treatment responsive; SZ, schizophrenia subjects; SZ-On, schizophrenia subject on medication; SZ-Off, schizophrenia subject off medication; TR, treatment resistant.

Demographics and other information. Some parameter information was not applicable for NCs. Therefore, an ANOVA was performed for parameters comparing three groups, and a $t$-test performed for parameters containing two groups. Pearson's $\chi^{2}$ test was used to compare categorical variables. 
vGLUT1, and vGLUT2. Western blot assays were performed as previously reported (Perez-Costas et al, 2012), with the following exceptions. Samples intended for vGLUT assays were not heated in order to avoid protein aggregation. Protein extracts $(60 \mu \mathrm{g})$ were loaded onto $4-20 \%$ gradient polyacrylamide gels (Lonza, Basel, Switzerland; 58505). Proteins were resolved by sodium dodecyl sulfatepolyacrylamide gel electrophoresis at $150 \mathrm{~V}$ for $1 \mathrm{~h} 15 \mathrm{~min}$, and then transferred at $30 \mathrm{~V}$ for $21 \mathrm{~h}$ onto polyvinylidene fluoride (PVDF) membranes (Bio-Rad; 162-0174) at $4{ }^{\circ} \mathrm{C}$. Initial analyses were performed to determine the optimal antibody concentrations. The following antibodies and concentrations were used: rabbit anti-vGLUT1 (1:1000, Mab Technologies (VGT1-3)); rabbit anti-vGLUT2 (1 : 8000, Synaptic Systems (135 403)); mouse anti-tyrosine hydroxylase (1:5000, Sigma (T2928)); mouse anti-GAD67 (1:1000, Millipore (MAB5406)); and mouse anti-actin $(1: 40000$, Millipore (MAB1501)). Each gel contained a mixture of NC and schizophrenia subjects, and was performed in duplicate. The membranes were blocked for $1 \mathrm{~h}$ in 5\% milk in Tris-buffered saline with Tween-20 (TBST), with the exception of the GAD67 assay that was blocked for $1 \mathrm{~h}$ in 3\% milk in TBST. Antigen presence was detected by incubating the primary antibody with the PVDF membrane for $21 \mathrm{~h}$ at $4^{\circ} \mathrm{C}$, with the exception of vGLUT1 that was incubated for $1 \mathrm{~h}$ at $4^{\circ} \mathrm{C}$. The bands were visualized using chemiluminescence (Bio-Rad; 170-5018), exposing Sigma-Aldrich Carestream Kodak BioMax XAR films (166-0760).

\section{Analyses}

Data. As described previously (McCollum et al, 2015), films were scanned at $600 \mathrm{dpi}$ using a flatbed scanner. Optical densities of the bands were measured using Image J-64 freeware $(\mathrm{NIH})$. A step calibration tablet was used in order to create an optical density standard curve (Stouffer Industries, Mishawaka, IN; T2120, series 130501) that each measurement was calibrated to. ImageJ was used to perform a background subtraction for each film. All optical density values for each protein were normalized to actin, and then to the averaged NC. These values were averaged for duplicate samples.

Statistics. Demographics and tissue quality were tested using ANOVA and/or $t$-tests. Categorical variables were assessed using Pearson's $\chi^{2}$ test. The data were tested for outliers using ROUT $(Q=1.0 \%)$ and Grubb's method $(\alpha=0.05)$ via Prism 6; no subjects qualified for removal using either method. The data were assessed for normality with both Shapiro-Wilk test and D'Agostini and Pearson test. If both tests revealed that the data were normally distributed, parametric tests were used (two groups, unpaired $t$-test; three groups, one-way ANOVA). If not, nonparametric tests were used (two groups, Mann-Whitney $U$-test; three groups, Kruskal-Wallis $H$-test). Significant omnibus tests were followed by a multiple comparisons test as suggested by Prism 6; parametric tests were followed by Holm-Sidak's test, whereas nonparametric tests were followed by Dunn's comparison. Furthermore, BrownForsythe and Bartlett's tests were used to detect the presence of significantly different SDs. Correlational analyses were performed between results and PMI, age, years frozen, and $\mathrm{pH}$ to elucidate potential relationships among these variables. Information was not present on enough subjects to test smoking status, tardive dyskinesia, or the deficit syndrome.

\section{RESULTS}

\section{Demographics}

The control (NC) and schizophrenia groups were well matched for age, race, sex, $\mathrm{PMI}, \mathrm{pH}$, and years frozen, and did not significantly differ. The demographics of the preliminary groups divided by treatment status or response also did not significantly differ (Table 1).

\section{Schizophrenia vs Normal Controls}

Schizophrenia subjects had elevated TH levels in comparison with NCs (an increase of 69.6\%, Figures 1a and b). Moreover, there was a wider spread of values in the SZ group compared with the NCs. To investigate this, correlational analyses were performed (Table 2). In the entire sample, $\mathrm{TH}$ levels were negatively correlated with age but positively correlated with PMI; however, there was no difference in these demographics between groups. Comparisons of correlation coefficients between groups for these variables were nonsignificant (Table 2). Thus, the bimodal distribution of $\mathrm{TH}$ in the SZ group is unlikely to be caused by the above variables. Schizophrenia subjects also exhibited higher GAD67 levels than NC subjects (an increase of 19.5\%; Figures $1 \mathrm{a}$ and $\mathrm{c}$ ). A correlation analysis of GAD67 protein with demographic variables revealed a negative correlation with age in the entire sample, but the effect was lost when comparing groups (Table 3). vGLUT1 and vGLUT2 levels were not significantly different between groups, but tended to be elevated in schizophrenia subjects (Figures 1a, d, and e).

Significant positive correlations were observed between TH and GAD67, and between vGLUT1 and vGLUT2 in the whole sample (Table 4). The significant relationship was maintained between $\mathrm{TH}$ and GAD67 in both groups (Table 4). However, there was no significant difference in these relationships between NC and schizophrenia subjects (Table 4). A negative correlation between vGLUT2 and $\mathrm{pH}$ was observed in the whole sample $(r=-0.464, P=0.03$; data not shown), but this relationship was lost between groups $(P>0.05)$. No other significant correlations with demographics were observed for vGLUT1 or vGLUT2 in the whole sample, NC, or SZ groups.

\section{Treatment Status: Effects of Antipsychotics}

SZ-On subjects exhibited significantly higher (an increase of 88.3\%) TH protein levels and GAD67 levels (an increase of $40.6 \%$ ) vs NCs (Figures 2a-c). In contrast, vGLUT2 levels were significantly elevated in the SZ-Off group (an increase of $28.7 \%$ ) in comparison with NCs (Figure 2e). No differences were observed for vGLUT1. Included in Supplementary Material is the analysis of treatment type (typical $v s$ atypical antipsychotic medication), which was nonsignificant, with both groups of subjects (typical and atypical treated groups) exhibiting significant increases in 
a

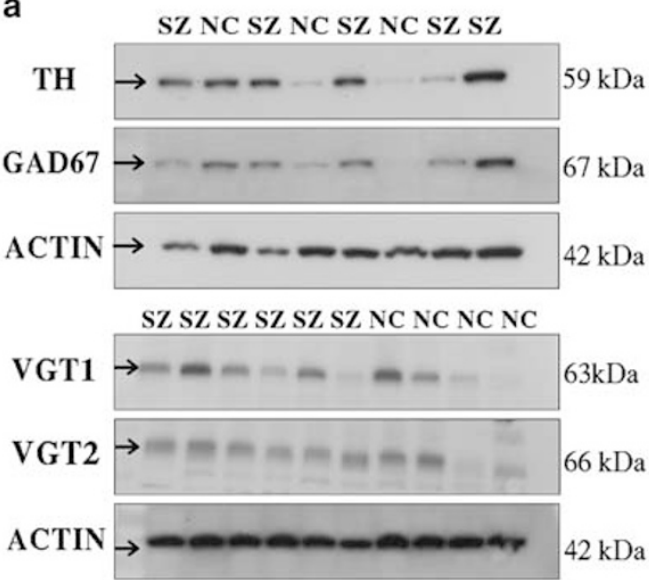

b

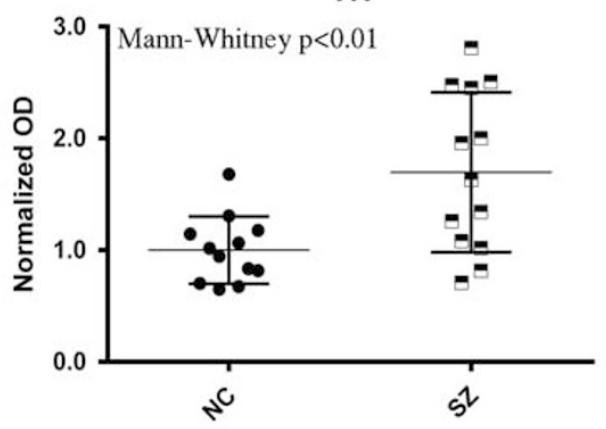

d

VGT1

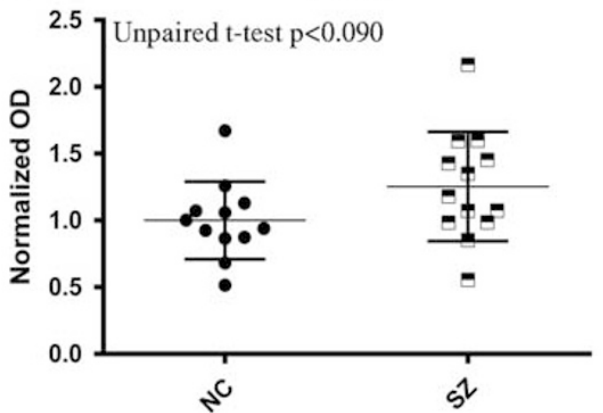

C GAD67

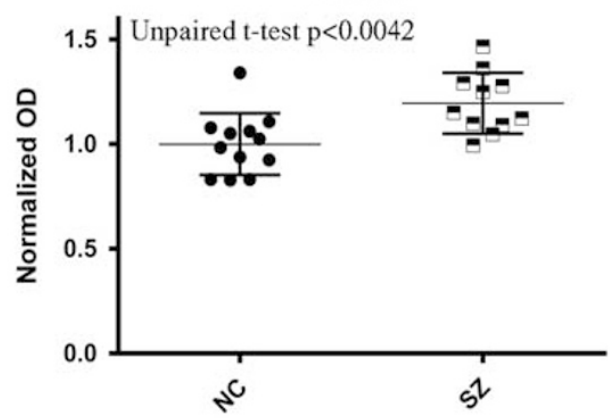

e

VGT2

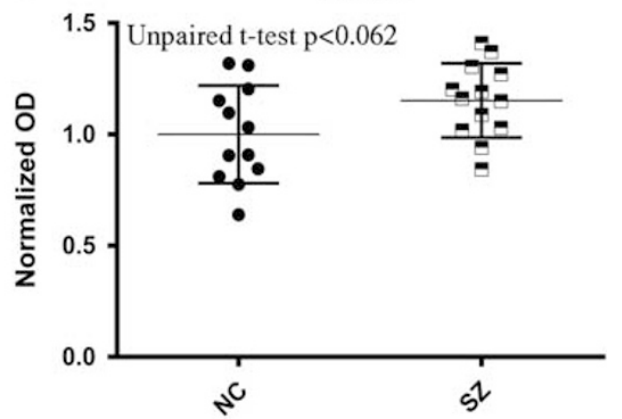

Figure I Controls vs schizophrenia. Representative western blots (a) and data are shown for TH (b), GAD67 (c), vGLUTI (d), and vGLUT2 (e) in normal controls (NCs) and schizophrenia (SZ). Arrows point to the primary band at the expected molecular weight of each respective protein that was analyzed. Actin was a loading control. Error bars represent SD. TH (SZ: $1.696 \pm 0.716$; NC: $1.000 \pm 0.300)$ was evaluated by Mann-Whitney $U$-test $(U=31$, $P=0.0096$ ). GAD67 (SZ: I.I $95 \pm 0.145$; NC: I.000 \pm 0.147 ) was evaluated by unpaired $t$-test $=t(2 \mid)=3.208, P=0.0042$. vGLUTI (SZ: I.254 $\pm 0.4 \mid 0 ;$ NC: $1.000 \pm 0.290 ; t(23)=1.772, P=0.0896)$ and $v G L U T 2$ (SZ: I.I52 $\pm 0.167 ; N C: 1.000 \pm 0.220 ; t(23)=1.963, P=0.06 \mid 8)$ levels were also evaluated by unpaired $t$-tests.

TH and GAD67 protein levels vs NCs (Supplementary Figure S1).

\section{Treatment Response}

TR subjects exhibited significantly higher (an increase of $121.0 \%$ ) TH protein levels than NCs (Figures $3 \mathrm{a}$ and $\mathrm{b}$ ). GAD67 levels were also significantly elevated (an increase of $58.7 \%$ ) in TR compared with NCs (Figures $3 a$ and c). No significant differences were observed for vGLUT1 or vGLUT2 (Figures 3a, d, and e).

\section{DISCUSSION}

Our main results suggest increased SN dopamine and GABA synthesis in schizophrenia compared with NCs as shown by elevated TH and GAD67 levels, respectively. Our preliminary analyses indicate similar elevations in DA and GABA synthesis in SZ-On and TR subjects in the context of treatment status and treatment response. Subcortical glutamate dysregulation is suggested by elevated vGLUT2 levels in the preliminary analysis of treatment status in SZ-Off subjects. Integration of data from the current study and that of previous findings is shown in Figure 4a. 
Table 2 Variables Correlated with TH Protein Levels

\begin{tabular}{lcccc}
\hline & WS & NC & SZ & CC \\
\hline No. & 25 & 12 & 13 & NC vs SZ \\
PMI & $P=\mathbf{0 . 0 3}$ & $P=0.35$ & $P=0.38$ & $P=0.88$ \\
& $r=\mathbf{0 . 4 5}$ & $r=0.33$ & $r=0.27$ & \\
Age & $P=\mathbf{0 . 0 3}$ & $P=0.67$ & $P=0.08$ & $P=0.37$ \\
& $r=-\mathbf{0 . 4 3}$ & $r=-0.14$ & $r=-0.50$ & \\
PH & $P=0.60$ & $P=0.35$ & $P=0.69$ & $P=0.64$ \\
& $r=0.12$ & $r=0.33$ & $r=0.13$ & \\
Years frozen & $P=0.60$ & $P=0.94$ & $P=0.87$ & $P=0.88$ \\
& $r=0.11$ & $r=0.02$ & $r=-0.05$ &
\end{tabular}

Abbreviations: CC, comparison of coefficients; NC, normal controls; PMI, postmortem interval; SZ, schizophrenia subjects; WS, whole sample.

Correlational analyses of demographic and tissue quality variables as they relate to TH protein levels. In the whole sample (WS), a positive correlation was observed between TH protein levels and PMl, whereas a negative relationship was observed between TH protein levels and age. These correlations were lost between groups. Significant $P$-values are shown in bold.

Table 3 Variables Correlated with GAD67 Protein Levels

\begin{tabular}{lcccc}
\hline & WS & NC & SZ & CC \\
\hline No. & 25 & 12 & 13 & NC vs SZ \\
PMI & $P=0.07$ & $P=0.11$ & $P=0.58$ & $P=0.36$ \\
& $r=0.38$ & $r=0.53$ & $r=0.17$ & \\
Age & $P=\mathbf{0 . 0 5}$ & $P=0.68$ & $P=0.14$ & $P=0.47$ \\
& $r=-\mathbf{0 . 4 0}$ & $r=-0.13$ & $r=-0.43$ & \\
$\mathrm{pH}$ & $P=0.58$ & $P=0.11$ & $P=0.81$ & $P=0.27$ \\
& $r=0.12$ & $r=0.53$ & $r=0.08$ & \\
Years frozen & $P=0.78$ & $P=0.90$ & $P=0.58$ & $P=0.65$ \\
& $r=0.06$ & $r=0.04$ & $r=-0.17$ & \\
\hline
\end{tabular}

Abbreviations: CC, comparison of coefficients; NC, normal controls; PMI, postmortem interval; SZ, schizophrenia subjects; WS, whole sample. Correlational analyses of demographic and tissue quality variables as they relate to GAD67 protein levels. A negative relationship was observed between GAD67 protein levels and age in the WS. Significant $P$-values are shown in bold.

\section{Limitations}

Our sample size is small, but not unusual for postmortem investigations (Rice et al, 2016; Howes et al, 2013; Mueller et al, 2004; Toru et al, 1988; Perez-Costas et al, 2012; Spokes et al, 1980). None of our subjects were first episode and antipsychotic naive, as is typical for postmortem studies. Thus, our results could potentially be affected by medication history.

\section{Dopamine and GABA Dysregulation in Schizophrenia}

Our results indicated elevated TH protein levels in the $\mathrm{SN}$ of schizophrenia subjects $v s$ NCs. Imaging studies have observed elevated dopamine synthesis in the SN (Howes et al, 2013) that has been supported by postmortem findings (Howes et al, 2013; Mueller et al, 2004; Toru et al, 1988), including the current study. Previously, we detected a decrease of $\mathrm{TH}$ protein and immunoreactivity in samples containing rostral SN/VTA (Perez-Costas et al, 2012;
Table 4 Correlations between Proteins

\begin{tabular}{|c|c|c|c|c|}
\hline & WS & NC & SZ & $\begin{array}{c}\mathrm{CC} \\
\mathrm{NC} \text { VS SZ }\end{array}$ \\
\hline TH/GAD67 & $\begin{array}{l}P=\mathbf{0 . 0 0 0 0 0 I} \\
r=\mathbf{0 . 8 0}\end{array}$ & $\begin{array}{l}P=\mathbf{0 . 0 0 0 4} \\
r=\mathbf{0 . 8 6}\end{array}$ & $\begin{array}{l}P=\mathbf{0 . 0 0 8} \\
r=\mathbf{0 . 7 0}\end{array}$ & $P=0.35$ \\
\hline TH/VGLUTI & $\begin{array}{l}P=0.25 \\
r=0.24\end{array}$ & $\begin{array}{l}P=0.18 \\
r=0.42\end{array}$ & $\begin{array}{l}P=0.95 \\
r=-0.02\end{array}$ & $P=0.38$ \\
\hline TH/VGLUT2 & $\begin{array}{l}P=0.22 \\
r=0.26\end{array}$ & $\begin{array}{l}P=0.37 \\
r=0.29\end{array}$ & $\begin{array}{l}P=0.92 \\
r=-0.03\end{array}$ & $P=0.47$ \\
\hline GAD67/VGLUTI & $\begin{array}{l}P=0.45 \\
r=0.16\end{array}$ & $\begin{array}{l}P=0.65 \\
r=0.15\end{array}$ & $\begin{array}{l}P=0.80 \\
r=-0.25\end{array}$ & $P=0.38$ \\
\hline GAD67/vGLUT2 & $\begin{array}{l}P=0.55 \\
r=0.13\end{array}$ & $\begin{array}{l}P=0.65 \\
r=0.15\end{array}$ & $\begin{array}{l}P=0.41 \\
r=-0.25\end{array}$ & $P=0.38$ \\
\hline vGLUTI/vGLUT2 & $\begin{array}{l}P=\mathbf{0 . 0 0 5} \\
r=\mathbf{0 . 5 5}\end{array}$ & $\begin{array}{l}P=0.08 \\
r=0.52\end{array}$ & $\begin{array}{l}P=0.09 \\
r=0.49\end{array}$ & $P=0.93$ \\
\hline
\end{tabular}

Abbreviations: CC, comparison of coefficients; NC, normal controls: PMI,

postmortem interval; SZ, schizophrenia subjects; WS, whole sample.

Correlational analyses between proteins. In the WS, a positive correlation was observed between TH and GAD67, and VGLUTI and VGLUT2. In NC and SZ groups, the positive correlation of TH/GAD67 was maintained, whereas the VGLUTI/VGLUT2 correlation was lost. Data are reported as mean and SD. Significant $P$-values are shown in bold.

Rice et al, 2016), whereas the present study used caudal SN in a different cohort of subjects (Figure $4 \mathrm{~b}$ ). Although the connectivity of the SN/VTA with its targets is somewhat intermingled (Williams and Goldman-Rakic, 1998; Haber and Fudge, 1997), mesocortical projections are derived heavily from the VTA (Porrino and Goldman-Rakic, 1982), whereas nigrostriatal projections are derived from the SNc (Fallon and Moore, 1978). The findings of decreased TH protein in rostral SN/VTA (Perez-Costas et al, 2012) and increased protein in caudal $\mathrm{SN}$ are therefore aligned with the hypodopaminergia observed in the frontal cortex (Akil et al, 1999; Slifstein et al, 2015) and the hyperdopaminergia in the striatum, respectively (Hietala et al, 1995; Howes and Kapur, 2009; Breier et al, 1997; Abi-Dargham et al, 1998; Laruelle et al, 1995, 1996). As the differences in TH levels could be due to different cohorts, a future study investigating $\mathrm{TH}$ levels in both regions of the same subjects is required for a definitive conclusion about regional differences in schizophrenia SN. TH levels in the schizophrenia group were bimodal (especially noticeable in Figure 2b), but the reason for this remains unclear as correlational analyses revealed no relationship between $\mathrm{TH}$ levels and demographics or tissue quality measures. Perhaps surprisingly to some, we observed a positive correlation between TH protein levels and PMI in the entire sample. Although the negative relationship between dopamine availability and postmortem interval is well known (Carlsson and Winblad, 1976), the effect of PMI on TH protein levels has not been examined. Booze et al (1993) provide the only known investigation of the effect of PMI on TH-staining axons; however, their study did not examine the $\mathrm{SN}$, and the changes they observed occurred in only $5 \%$ and $2 \%$ of $\mathrm{TH}$-staining axons, respectively (Booze et al, 1993). The positive correlation observed here between PMI and TH protein levels is not easily explained, but does not significantly change with group, and therefore should posit no negative effects on the results reported here. Expectedly, we also observed a negative relationship between 
a
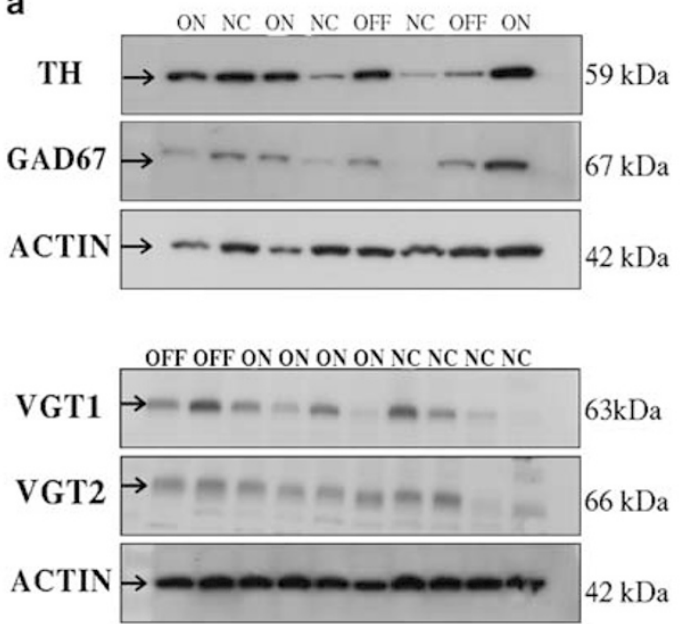

b

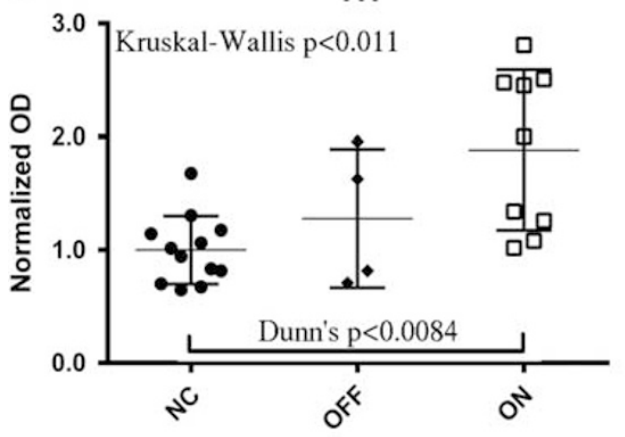

d

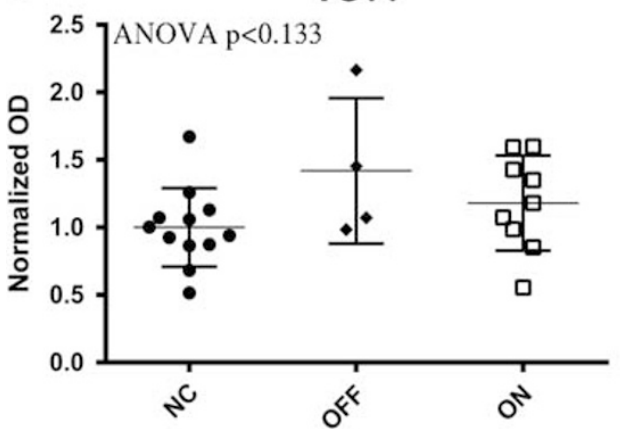

C

GAD67

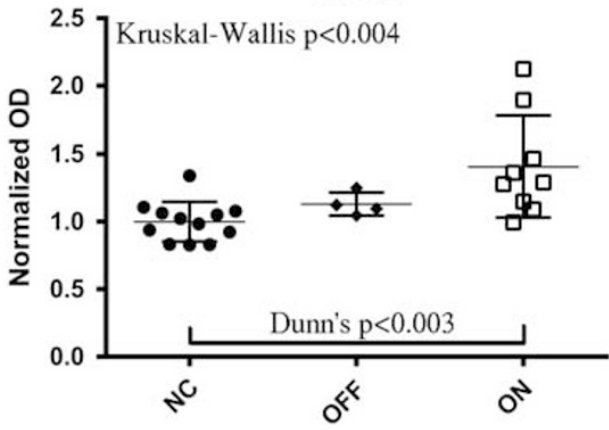

e

VGT2

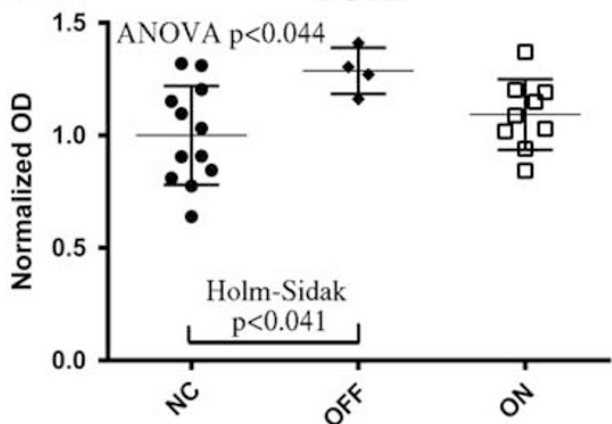

Figure 2 Controls vs Schizophrenia off or on medication. Representative western blots (a) and data are shown for TH (b), GAD67 (c), vGLUTI (d), and VGLUT2 (e) in normal controls (NC), OFF (unmedicated SZ), and ON (medicated SZ). Arrows point to the primary band at the expected molecular weight of each respective protein that was analyzed. Actin was a loading control. Error bars represent SD. TH levels were different among groups (Kruskal-Wallis $H$-test $(H(2)=9.061, P=0.0108)$, with a difference in TH levels between SZ-ON $(1.883 \pm 0.708)$ vs NCs $(1.000 \pm 0.300 ; D u n n ' s$ test, $P=0.0084)$. GAD67 protein levels were different among groups (Kruskal-Wallis $H$-test $H(2)=11.05, P=0.004$ ) with a difference in GAD67 levels between SZ-ON $(1.406 \pm 0.375)$ vs NCs $(1.000 \pm 0.147$; Dunn's test, $P=0.0029)$. Although no group differences were seen with $v G L U T I(F(2,22)=2.212, P=0.133)$, VGLUT2 levels were different among groups (ANOVA, $F(2,22)=3.612, P=0.044)$, being greater in the SZ-OFF $(1.287 \pm 0.102)$ than NC $(1.000 \pm 0.220$; Holm-Sidak, $P=0.041$ ).

both TH and GAD67 and age in the entire sample, offering further support for a disease-independent, age-related decline in both of these neurotransmitters.

SN hyperactivity, as well as increased markers of glutamate (White et al, 2015), is present in schizophrenia patients and correlated with psychosis regardless of whether they are medication naive or chronically medicated (Yoon et al, 2013, 2014). Possible mechanisms underlying hyperexcitability in the SN could be increased glutamate and/or decreased GABA levels. However, there were no statistically significant elevations in either vGLUT protein, nor was there a correlation between either vGLUT or TH. Furthermore, we observed an increase in GAD67 levels, along with a positive correlation of GAD67 with TH. These data suggest increases in GABA production in the SN in schizophrenia. There is a paucity of literature on GAD levels in the $\mathrm{SN}$ in schizophrenia; the only other study conducted found no abnormalities (Spokes et al, 1980). GAD67 is normally found 
a
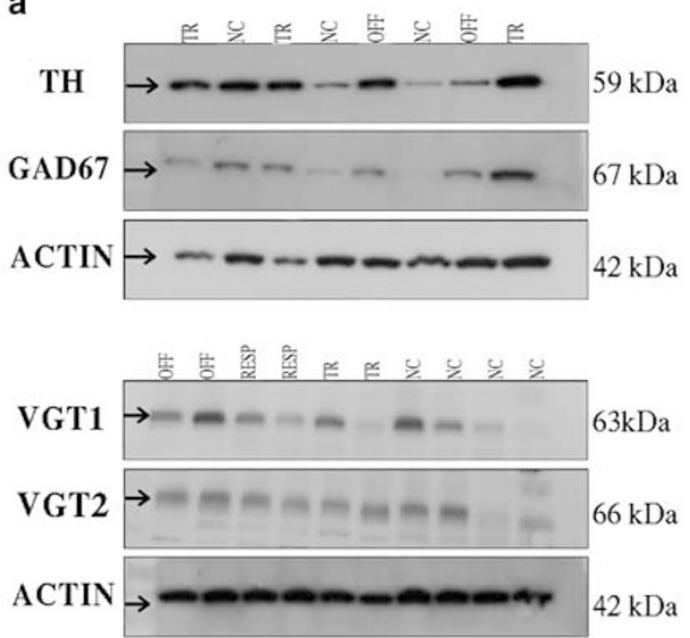

b

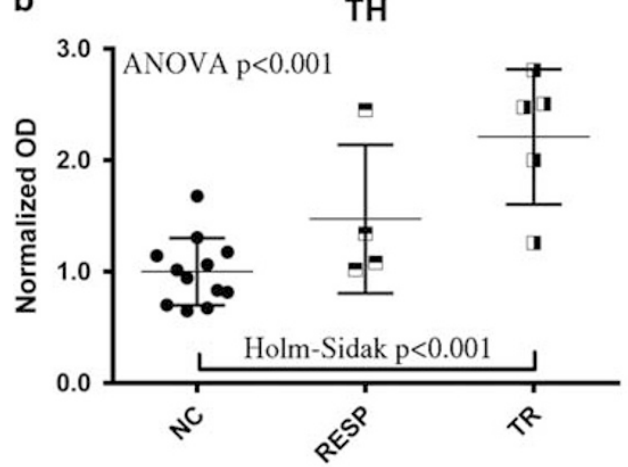

d

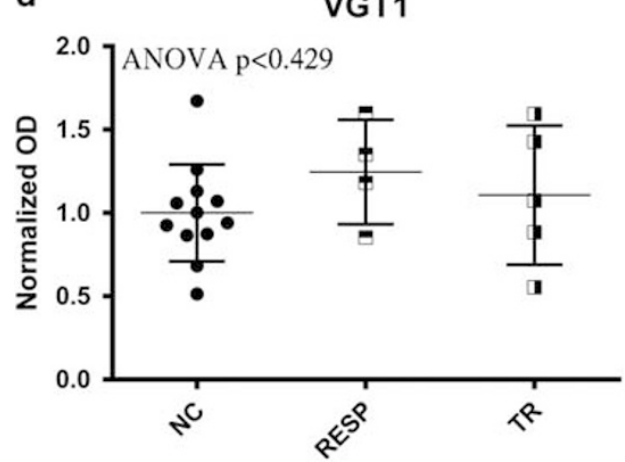

C

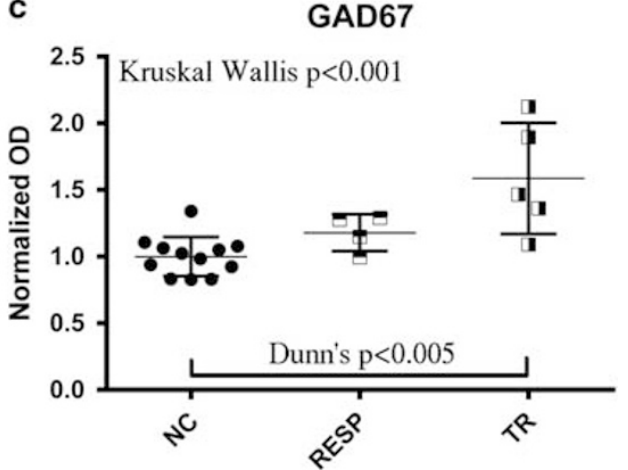

e

VGT2

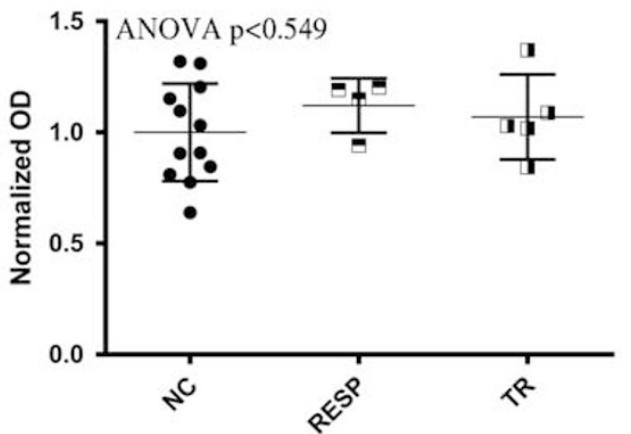

Figure 3 Controls vs schizophrenia treatment resistant (TR) or responsive (RESP). Representative western blots (a) and data are shown for TH (b), GAD67 (c), vGLUTI (d), and VGLUT2 (e) in normal controls (NC), RESP, and TR. Arrows point to the primary band at the expected molecular weight of each respective protein that was analyzed. Actin was a loading control. Error bars represent SD. TH levels were significantly different among groups (ANOVA, $F(2, \mid 8)=12.37, P=0.0004)$, with higher TH levels in TR $(2.210 \pm 0.606)$ than NC $(1.000 \pm 0.3000$; Holm-Sidak, $P=0.0003)$. GAD67 protein levels were different among groups (Kruskal-Wallis, $(H(2)=10.78, P=0.0009)$, with higher levels in TR $(1.587 \pm 0.417)$ vs NCs $(1.000 \pm 0.147$; Dunn's test, $P=0.004)$. No group differences were seen with vGLUTI $(F(2, \mid 8)=0.889, P=0.428)$ or vGLUT2 protein levels $(F(2, \mid 8)=0.6204, P=0.549)$.

in both cell bodies (Phelps et al, 1999) and terminals (Esclapez et al, 1994). Our results suggest an elevation of GABA synthesis in the SN of schizophrenia subjects, but the precise location of the abnormalities (soma vs terminals) is unknown. Elevated GAD67 levels could result from increased density or size of afferent GABAergic terminals, and/or increased protein synthesis within SN reticulata neurons and their intrinsic collaterals. Sources of GABAergic afferents to the $\mathrm{SN}$ include neurons that comprise the striatal direct pathway and neurons in the globus pallidus externus
(Haber and Fudge, 1997). Schizophrenia subjects exhibit increased striatal glutamate tone (de la Fuente-Sandoval et al, 2011, 2013) and number of glutamatergic synapses in comparison with NCs (Roberts et al, 2012; McCollum et al, 2015; Figure 4a). It is possible that the extra stimulation provided by the glutamatergic input onto the GABAergic striatal neurons produces an increase in GAD and GABA in the SN. Alternatively, the observed increase in GAD may stem from increased GABA in neurons in the $\mathrm{SN}$ reticulata (Figure 4a). 
a

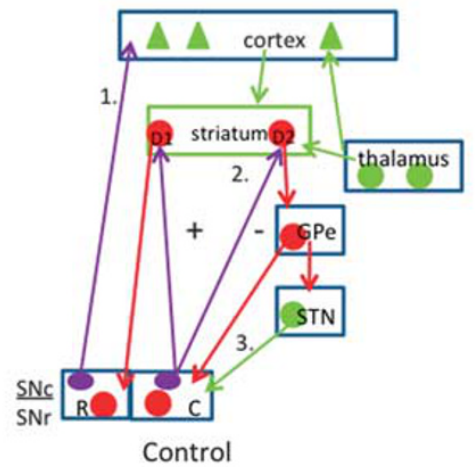

b

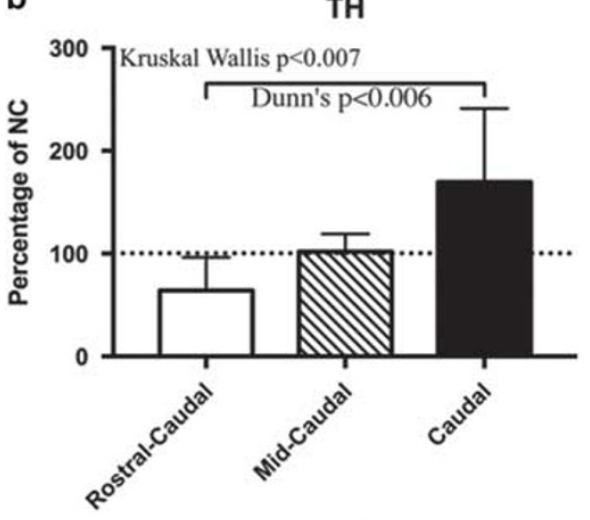

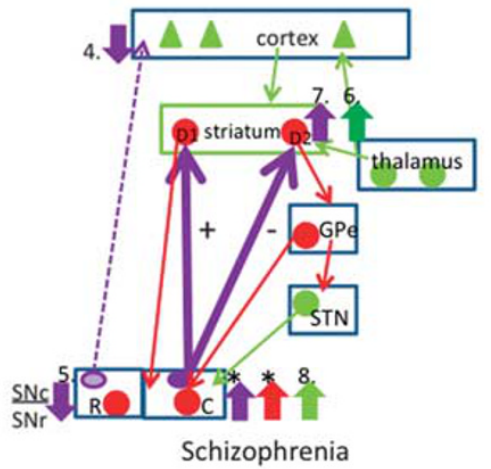

C

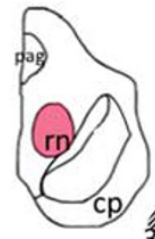

3n

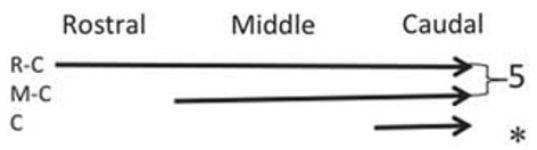

\section{SN Region}

Figure 4 (a) Diagram of nigral connections in normal controls and subjects with schizophrenia. Connections are shown by arrows: green for excitatory, red for inhibitory, and purple for dopamine. SNc, substantia nigra pars compacta; SNr, substantia nigra pars reticulata; R, rostral; C, caudal; STN, subthalamic nucleus; GPe, globus pallidus external segment; $D_{1}$, dopaminergic $D_{1}$ receptor; $D_{2}$, dopaminergic $D_{2}$ receptor. For reviews of basal ganglia circuitry, see PerezCostas et al (20 I0) and Haber and Fudge (1997); key references are cited: (I) Gaspar et al (1992); (2) Fallon and Moore (1978); (3) Lavoie and Parent (I 990); (4) Akil et al (1999) and Slifstein et al (20 I5); (5) Rice et al (2016) and Perez-Costas et al (20 I2); (6) de la Fuente-Sandoval et al (20II, 20 I3) and Roberts et al (2012); (7) Hietala et al (1995), Roberts et al (2009), McCollum et al (2015), Breier et al (1997), Abi-Dargham et al (I998), and Laruelle et al (1995, 1996); and (8) White et al (2015); * present study, 20 16. (b) SZ TH protein levels as percentage of NC TH protein levels in three regions of substantia nigra. Rostral-caudal and mid-caudal data are from our previous paper (Perez-Costas et al, 20 I2), whereas caudal data are from the current study. As suggested here, our data align with the often-replicated hypodopaminergia of the cortex and the hyperdopaminergia of the striatum. The rostral portion of the SN, responsible for mesocortical projections, is the locus of decreased TH levels; the caudal SN, responsible for the nigrostriatal pathway, is the locus of increased $\mathrm{TH}$ levels. Sections containing middle through caudal SN do not show a difference. Therefore, it is of keen importance when studying the substantia nigra to keep these anatomical differences in mind while planning, executing, and interpreting experiments. (c) Images of different rostrocaudal levels of the SN. pag, periaqueductal gray; $m$, red nucleus; $\mathrm{cp}$, cerebral peduncle; $3 n$, third nerve rootlets. The black box on the caudal section represents the block from which the current study's tissue was retrieved. The arrows below the three sections of SN represent the extent of the SN studied in our previous and current study.

\section{Preliminary Analysis of Treatment Status and Response}

Although sample size limits these analyses to exploratory data, we observed interesting results that merit exploration in larger cohorts.

\section{Treatment Status}

$\mathrm{TH}$ and GAD protein levels were elevated in medicated patients, whereas vGLUT2 levels were elevated in unmedicated patients. Although counterintuitive, the observed increases here in caudal but not in previously examined rostral SN TH protein (Perez-Costas et al, 2012) in medicated patients could actually be attributed to medication and its location of action. Both acute and chronic dopaminergic $\mathrm{D}_{2}$ receptor blockade of the striatum (the location of action of most antipsychotics) result in an increase in dopamine synthesis (Carlsson, 1974; Moghaddam and Bunney, 1990; Chertkow et al, 2007) and release in haloperidol-treated rats (Chertkow et al, 2007; Cobb and Abercrombie, 2002; Osborne et al, 1994). These findings indicate that the antipsychotic effects of medication are due to antagonism of striatal $\mathrm{D}_{2}$ receptors and not an overall decrease in dopaminergic synthesis or release. Therefore, a blockade of striatal $\mathrm{D}_{2}$ receptors would result in an increase of dopamine synthesis in the area of $\mathrm{SN}$ responsible for striatal projections, in addition to an increased release of dopamine from the terminals of the nigrostriatal pathway, as a compensatory mechanism to overcome the dopaminergic antagonism of the striatum. In addition, the cortex contains primarily $\mathrm{D}_{1}$ receptors with very few $\mathrm{D}_{2}$ receptors. Therefore, we would not expect to observe antipsychotic-increased $\mathrm{TH}$ levels in rostral SN (responsible for cortical projections), even in subjects on medication at the time of death as in our previous paper (Perez-Costas et al, 2012), as the cortex is not a target of antipsychotic-induced $\mathrm{D}_{2}$ antagonism. 
The current exploratory results of increased GAD67 in SZ-On subjects are supported by previous studies. Striatal $\mathrm{D}_{2}$ receptor stimulation results in a decreased release of GABA (Girault et al, 1986; Centonze et al, 2002), leading to speculation that $D_{2}$ receptor antagonism via medication could result in increased GABA release, as observed in the current preliminary analysis. Indeed, antipsychotic treatment in rats induces elevation of GABAergic activity and release in the striatum (Osborne et al, 1994).

Elevated striatal glutamate levels of first-episode, drugnaive subjects are normalized after a successful medication regimen (de la Fuente-Sandoval et al, 2013). Glutamate-like synapses are more numerous in SZ-Off and are decreased in number with successful treatment (Roberts et al, 2012) and with antipsychotic medication in rats (Roberts et al, 1995), suggesting that antipsychotic medication decreases glutamatergic tone, at least in striatum. Our exploratory finding of elevated vGLUT2 (indicative of increased subcortical glutamate release) (Raju et al, 2006) in SZ-Off, and not SZ-On, subjects are consistent with previous findings of medicationinduced normalization of glutamate release in other brain regions.

\section{Treatment Response}

Approximately $30 \%$ of medicated patients do not respond to treatment (Conley and Kelly, 2001). Imaging studies reveal that TR subjects show normal or subnormal striatal DA synthesis capacity (Demjaha et al, 2012). However, very few studies have been conducted using postmortem tissue to investigate treatment resistance (Roberts et al, 2009, 2012), and to date, none have examined the role of the $\mathrm{SN}$ in treatment response.

We observed elevated TH protein levels in TR subjects in comparison with NCs, suggesting elevated DA synthesis. These investigatory results appear in contrast with previous literature conducted in the striatum that observed similar DA synthesis in TR subjects and NCs (Demjaha et al, 2012). However, a potential explanation for this may be abnormal anterograde transport of nigrostriatal dopamine synthesis machinery in TR subjects, resulting in a collection of excess $\mathrm{TH}$ in the $\mathrm{SN}$ that is unable to be transported to the striatum.

Our preliminary data suggest elevated nigral GABA synthesis in TR subjects when compared with NCs, but not against their responsive counterparts. Previous literature observed no change in SN GAD67 levels in schizophrenia subjects (Spokes et al, 1980), but did not investigate treatment response.

\section{CONCLUSIONS}

Our findings suggest abnormality of the dopamine and GABAergic systems in the SN in schizophrenia, with potential alterations in the glutamatergic system. This study provides insight into the rarely studied SN in schizophrenia, and offers the first preliminary data on treatment status and response. These results highlight potential abnormalities of dopaminergic, GABAergic, and glutamatergic interaction. Future directions include but are not limited to immunoblotting, immunohistochemistry, and ultrastructural analysis of cytoskeletal structure in major white matter tracts connecting the substantia nigra with affected brain regions in schizophrenia.

\section{FUNDING AND DISCLOSURE}

The authors declare no conflict of interest.

\section{ACKNOWLEDGMENTS}

We thank the staff of the Maryland Brain Collection for the samples used in this study. We also thank Dr Miguel Melendez-Ferro for sectioning the brain tissue. This work was supported by the National Institute of Mental Health (F31MH098566 to LAM and RO1MH066123 to RCR).

\section{REFERENCES}

Abi-Dargham A, Gil R, Krystal J, Baldwin R, Seibyl J, Bowers M et al (1998). Increased striatal dopamine transmission in schizophrenia: confirmation in a second cohort. Am J Psychiatry 155: 761-767.

Akil M, Pierri JN, Whitehead RE, Edgar CL, Mohila C, Sampson AR et al (1999). Lamina-specific alterations in the dopamine innervation of the prefrontal cortex in schizophrenic subjects. Am J Psychiatry 156: 1580-1589.

Bellocchio E, Reimer R, Fremeau R Jr, Edwards RH (2000). Uptake of glutamate into synaptic vesicles by an inorganic phosphate transporter. Science 289: 957-960.

Booze RM, Mactutus CF, Gutman CR, Davis JN (1993). Frequency analysis of catecholamine axonal morphology in human brain. J Neurol Sci 119: 99-109.

Breier A, Su T, Saunders R, Carson RE, Kolachana BS, de Bartolomeis A et al (1997). Schizophrenia is associated with elevated amphetamine-induced synaptic dopamine concentrations: evidence from a novel positron emission tomography method. Proc Natl Acad Sci USA 94: 2569-2574.

Carlsson A (1974). Antipsychotic drugs and catecholamine synapses. J Psychiatr Res 11: 57-64.

Carlsson A, Lindqvist M (1963). Effect of chlorpromazine and haloperidol on formation of 3-methoxytyramine and normetanephrine in mouse brain. Acta Pharmacol Toxicol (Copenh) 20: 140-144.

Carlsson A, Winblad B (1976). Influence of age and time interval between death and autopsy on dopamine and 3-methoxytyramine levels in human basal ganglia. J Neural Transm 38: 271-276.

Centonze D, Picconi B, Baunez C, Borrelli E, Pisani A, Bernardi G et al (2002). Cocaine and amphetamine depress striatal GABAergic synaptic transmission through D2 dopamine receptors. Neuropsychopharmacology 26: 164-175.

Chertkow Y, Weinreb O, Youdim MB, Silver H (2007). Dopamine and serotonin metabolism in response to chronic administration of fluvoxamine and haloperidol combined treatment. J Neural Transm (Vienna) 114: 1443-1454.

Cobb W, Abercrombie E (2002). Distinct roles for nigral GABA and glutamate receptors in the regulation of dendritic dopamine release under normal conditions and in response to systemic haloperidol. J Neurosci 22: 1407-1413.

Conley R, Kelly D (2001). Management of treatment resistance in schizophrenia. Biol Psychiatry 50: 898-911.

Creese I, Burt DR, Snyder SH (1976). Dopamine receptors and average clinical doses. Science 194: 546.

Damier P, Hirsch EC, Agid Y, Graybiel AM (1999). The substantia nigra of the human brain. I: Nigrosome and the nigral matrix, a 
compartmental organization based on calbindin $\mathrm{D}_{28 \mathrm{~K}}$ immunohistochemistry. Brain 122: 1421-1436.

Delay J, Deniker P, Harl JM, Grasset A (1952). N-dimethylaminoprophylchlorophenothiazine (4560 RP) therapy of confusional states. Ann Méd Psychol (Paris) 110: 398-403.

de la Fuente-Sandoval C, Leon-Ortiz P, Azcarraga M, Stephano S, Favila R, Diaz-Galvis L et al (2013). Glutamate levels in the associative striatum before and after 4 weeks of antipsychotic treatment in first-episode psychosis: a longitudinal proton magnetic resonance spectroscopy study. JAMA Psychiatry 70: 1057-1066.

de la Fuente-Sandoval C, Leon-Ortiz P, Favila R, Stephano S, Mamo D, Ramirez-Bermudez J et al (2011). Higher levels of glutamate in the associative-striatum of subjects with prodromal symptoms of schizophrenia and patients with first-episode psychosis. Neuropsychopharmacology 36: 1781-1791.

Demjaha A, Murray RM, McGuire PK, Kapur S, Howes OD (2012). Dopamine synthesis capacity in patients with treatment-resistant schizophrenia. Am J Psychiatry 169: 1203-1210.

Esclapez M, Tillakaratne NJ, Kaufman DL, Tobin AJ, Houser CR (1994). Comparative localization of two forms of glutamic acid decarboxylase and their mRNAs in rat brain supports the concept of functional differences between the forms. J Neurosci 14: 1834-1855.

Fallon J, Moore R (1978). Catecholamine innervation of the basal forebrain IV: topography of the dopamine projection to the basal forebrain and neostriatum. J Comp Neurol 180: 545-580.

Gaspar P, Stepniewska I, Kaas JH (1992). Topography and collateralization of the dopaminergic projections to motor and lateral prefrontal cortex in owl monkeys. J Comp Neurol 325: $1-21$.

Girault J, Spampinato U, Glowinski J, Besson MJ (1986). In vivo release of $[3 \mathrm{H}] \gamma$-aminobutyric acid in the rat neostriatum-II. Opposing effects of D1 and D2 dopamine receptor stimulation in the dorsal caudate putamen. Neuroscience 9: 1109-1117.

Haber S, Fudge JL (1997). The primate substantia nigra and VTA: integrative circuitry and function. Crit Rev Neurobiol 11: 323-342.

Hadley J, Nenert R, Kraguljac NV, Bolding MS, White DM, Skidmore FM et al (2014). Ventral tegmental area/midbrain functional connectivity and response to antipsychotic medication in schizophrenia. Neuropsychopharmacology 39: 1020-1030.

Hall H, Reyes S, Landeck N, Bye C, Leanza G, Double K et al (2014). Hippocampal Lewy pathology and cholinergic dysfunction are associated with dementia in Parkinson's disease. Brain 137: 2493-2508.

Hietala J, Syvalahti E, Vuorio K, Rakkolainen V, Bergman J, Haaparanta $M$ et al (1995). Presynaptic dopamine function in striatum of neuroleptic-naive schizophrenic patients. Lancet 346: 1130-1131.

Howes O, Kapur S (2009). The dopamine hypothesis of schizophrenia: version III- the final common pathway. Schizophr Bull 35: 549-562.

Howes O, Williams M, Ibrahim K, Leung G, Egerton A, McGuire PK et al (2013). Midbrain dopamine function in schizophrenia and depression: a post-mortem and positron emission tomographic imaging study. Brain 136: 3242-4251.

Ichinose $\mathrm{H}$, Ohye T, Fujita K, Pantucek F, Lange K, Riderer P et al (1994). Quantification of mRNA of tyrosine hydroxylase and aromatic L-amino acid decarboxylase in the substantia nigra in Parkinson's disease and schizophrenia. J Neural Transm Park Dis Dement Sect 8: 149-158.

Kane JM, Honigeld G, Singer J, Meltzer H (1988). Clozapine in treatment-resistant schizophrenics. Psychopharmacol Bull 24: 62-67.

Laruelle M, Abi-Dargham A, van Dyck CH, Gil R, D'Souza CD, Erdos J et al (1996). Single photon emission computerized tomography imaging of amphetamine-induced dopamine release in drug-free schizophrenic subjects. Proc Natl Acad Sci USA 93: 9235-9249.

Laruelle M, Abi-Dargham A, van Dyck CH, Rosenblatt W, ZeaPonce Y, Zoghbi SS et al (1995). SPECT imaging of striatal dopamine release after amphetamine challenge. J Nucl Med 36: 1182-1190.

Lavoie B, Parent A (1990). Immunohistochemical study of the serotoninergic innervation of the basal ganglia in the squirrel monkey. J Comp Neurol 299: 1-16.

McCollum L, Walker CK, Roche JK, Roberts RC (2015). Elevated excitatory input to the nucleus accumbens in schizophrenia: a postmortem ultrastructural study. Schizophr Bull 41: 1123-1132.

Meyer-Lindenberg A, Miletich RS, Kohn PD, Esposito G (2002). Reduced prefrontal activity predicts exaggerated striatal dopaminergic function in schizophrenia. Nat Neurosci 5: 267-271.

Moghaddam B, Bunney B (1990). Acute effects of typical and atypical antipsychotic drugs on the release of dopamine from prefrontal cortex, nucleus accumbens, and striatum of the rat: an in vivo microdialysis study. J Neurochem 54: 755-760.

Mueller T, Haroutunian V, Davis KL, Meador-Woodruff JH (2004). Expression of the ionotropic glutamate receptor subunits and NMDA receptor-associated intracellular proteins in the substantia nigra in schizophrenia. Mol Brain Res 121: 60-69.

Nagatsu T, Levitt M, Udenfriend S (1964). Tyrosine hydroxylase. The initial step in norepinephrine biosynthesis. J Biol Chem 239: 2910-2917.

Osborne P, O'Connor WT, Beck O, Ungerstedt U (1994). Acute versus chronic haloperidol: relationship between tolerance to catalepsy and striatal and accumbens dopamine, GABA, and acetylcholine release. Brain Res 634: 20-30.

Perez-Costas E, Melendez-Ferro M, Rice MW, Conley RR, Roberts RC (2012). Dopamine pathology in schizophrenia: analysis of total and phosphorylated tyrosine hydroxylase in the substantia nigra. Front Psychiatry 9: 31.

Perez-Costas E, Melendez-Ferro M, Roberts RC (2010). Basal ganglia pathology in schizophrenia: dopamine connections and anomalies. J Neurochem 113: 287-302.

Phelps P, Alijani A, Tran T (1999). Ventrally located commissural neurons express the GABAergic phenotype in developing rat spinal cord. J Comp Neurol 409: 285-298.

Porrino L, Goldman-Rakic PS (1982). Brainstem innervation of prefrontal and anterior cingulate cortex in the rhesus monkey revealed by retrograde transport of HRP. J Comp Neurol 205: 63-76.

Raju DV, Shah DJ, Wright TM, Hall RA, Smith Y (2006). Differential synaptology of vGluT2-containing thalamostriatal afferents between the patch and matrix compartments in rats. J Comp Neurol 499: 231-243.

Rice M, Roberts RC, Melendez-Ferro M, Perez-Costas E (2016). Mapping dopaminergic deficiencies in the substantia nigra/ ventral tegmental area in schizophrenia. Brain Struct Funct 221: 185-201.

Roberts RC, Gaither LA, Gao XM, Kashyap SM, Tamminga CA (1995). Ultrastructural correlates of haloperidol-induced oral dyskinesias in rat striatum. Synapse 20: 234-243.

Roberts R, Roche JK, Conley RR, Lahti AC (2009). Dopaminergic synapses in the caudate of subjects with schizophrenia: relationship to treatment response. Synapse 63: 520-530.

Roberts RC, Roche JK, Somerville SM, Conley RR (2012) Ultrastructural distinctions between treatment responders and non-responders in schizophrenia: postmortem studies of the striatum. In:Labate L (ed). Mental Illnesses - Evaluation, Treatments and Implications. InTech: Croatia. pp 261-286.

Schoonover KS, McCollum LA, Roberts RC (2015). Tyrosine hydroxylase, GAD67, vGLUT1, and vGLUT2 proteins in the substantia nigra in schizophrenia. Poster presented at the Society for Neuroscience annual meeting, Chicago, IL. 
Slifstein M, van de Giessen E, Van Snellenberg J, Thompson JL, Narendran R, Gil R et al (2015). Deficits in prefrontal cortical and extrastriatal dopamine release in schizophrenia: a positron emission tomographic functional magnetic resonance imaging study. JAMA Psychiatry 72: 316-324.

Spokes E, Garrett NJ, Rossor MN, Iverson LL (1980). Distribution of GABA in post-mortem brain tissue from control, psychotic, and Huntington's chorea subjects. J Neurol Sci 48: 303-313.

Stan AD, Ghose S, Gao XM, Roberts RC, Lewis-Amezcua K, Hatanpaa KJ et al (2006). Human postmortem tissue: what quality markers matter? Brain Res 1123: 1-11.

Storm-Mathisen J (1974). GABA as a transmitter in the central nervous system of vertebrates. J Neural Transm 11: 227-253.

Toru M, Watanabe S, Shibuya H, Nishikawa T, Noda K, Mitsushio H et al (1988). Neurotransmitters, receptors, and neuropeptides in post-mortem brains of chronic schizophrenic patients. Acta Psychiatr Scand 78: 121-137.

Watanabe Y, Tanaka A, Tsukabe A, Kunitomi Y, Nishizawa M, Hashimoto $\mathrm{R}$ et al (2014). Neuromelanin magnetic resonance imaging reveals increased dopaminergic neuron activity in the substantia nigra of patients with schizophrenia. PLoS One 9: e104619.

White DM, Kraguljac NV, Reid MA, Lahti AC (2015). Contribution of substantia nigra glutamate to prediction error signals in schizophrenia: a combined magnetic resonance spectroscopy/ functional imaging study. NPJ Schizophr 1: 14001.

Williams SM, Goldman-Rakic PS (1998). Widespread origin of the primate mesofrontal dopamine system. Cereb Cortex 8: 321-345.

Yoon JH, Minzenberg MJ, Raouf S, D’Esposito M, Carter CS (2013). Impaired prefrontal-basal ganglia functional connectivity and substantia nigra hyperactivity in schizophrenia. Biol Psychiatry 74: $122-129$.

Yoon JH, Westphal AJ, Minzenberg MJ, Niendam T, Ragland D, Lesh $\mathrm{T}$ et al (2014). Task-evoked substantia nigra associated with prefrontal hypofunction, prefrontonigral disconnectivity and nigrostriatal connectivity predicting psychosis severity in medication naïve first episode schizophrenia. Schizophr Res 159: 521-526.

Supplementary Information accompanies the paper on the Neuropsychopharmacology website (http://www.nature.com/npp) 